\title{
THE ROLE OF PRESUMED PROBABILITY DENSITY FUNCTIONS IN THE SIMULATION OF NONPREMIXED TURBULENT COMBUSTION
}

\author{
A. Coclite ${ }^{1,2}$, G. Pascazio ${ }^{1,2}$, P. De Palma ${ }^{1,2}$, \\ and L. Cutrone ${ }^{2,3}$ \\ ${ }^{1}$ Dipartimento di Meccanica, Matematica e Management \\ 200 Via Re David, Bari 70125, Italy \\ ${ }^{2}$ Centro di Eccellenza in Meccanica Computazionale (CEMEC) \\ 200 Via Re David, Bari 70125, Italy \\ ${ }^{3}$ Centro Italiano di Ricerche Aerospaziali (CIRA) \\ Via Maiorise, Capua 81043, Italy
}

\begin{abstract}
Flamelet-Progress-Variable (FPV) combustion models allow the evaluation of all thermochemical quantities in a reacting flow by computing only the mixture fraction $Z$ and a progress variable $C$. When using such a method to predict turbulent combustion in conjunction with a turbulence model, a probability density function (PDF) is required to evaluate statistical averages (e.g., Favre averages) of chemical quantities. The choice of the PDF is a compromise between computational costs and accuracy level. The aim of this paper is to investigate the influence of the PDF choice and its modeling aspects to predict turbulent combustion. Three different models are considered: the standard one, based on the choice of a $\beta$-distribution for $Z$ and a Dirac-distribution for $C$; a model employing a $\beta$-distribution for both $Z$ and $C$; and the third model obtained using a $\beta$-distribution for $Z$ and the statistically most likely distribution (SMLD) for $C$. The standard model, although widely used, does not take into account the interaction between turbulence and chemical kinetics as well as the dependence of the progress variable not only on its mean but also on its variance. The SMLD approach establishes a systematic framework to incorporate informations from an arbitrary number of moments, thus providing an improvement over conventionally employed presumed PDF closure models. The rational behind the choice of the three PDFs is described in some details and the prediction capability of the corresponding models is tested vs. well-known test cases, namely, the Sandia flames, and $\mathrm{H}_{2}$-air supersonic combustion.
\end{abstract}




\section{INTRODUCTION}

The industrial and scientific communities devote major research efforts to identify and assess innovative technologies for advanced propulsive concepts. Among such technologies, hydrocarbon combustion has been assumed as a key issue to achieve better propulsive performance and lower environmental impact. To improve the know-how to build more efficient engines with lower emissions, it is necessary to enhance the knowledge of the combustion phenomena. In this context, the simulation of turbulent reacting flows is very useful to cut down experimental costs and to achieve a thorough comprehension of the physical mechanisms involved. Turbulent combustion is a multiscale problem, where the interaction between chemical kinetics, molecular, and turbulent transport occurs over a wide range of length and time scales. The numerical simulation of such phenomena with detailed chemistry is today prohibitive, so that a reduction model is often employed to compress the reaction mechanisms and cut down the computational costs. Different approaches have been proposed to address this problem such as the reduction of the chemical scheme in intrinsic low-dimensional manifolds (ILDM) [1]; the flamelet-based approaches such as the FPV [2] or flame prolongation of ILDM (FPI) [3]; and the flamelet-generated manifolds (FGM) approach [4].

The present authors' interest is devoted here to diffusive, either partially premixed or nonpremixed, flames which constitute a specific class of combustion problems where fuel and oxidizer are not mixed before they enter the combustion chamber. In this case, mixing must bring reactants into the reaction zone so as to activate and maintain the combustion process. Nonpremixed flames can be characterized by a local balance between diffusion and reaction [5]. Their structure can be described by a conserved scalar, the so-called mixture fraction. A diffusive flame can be viewed as an ensemble of thin locally one-dimensional structures embedded within the flow field. Each element of the flame front can then be described as a small laminar flame, also called flamelet. In this paper, the authors focus on the FPV approach for turbulent nonpremixed flames. The FPV approach is based on the use of only two degrees of freedom, namely, the mixture fraction and the progress variable, that are employed to map all of the thermochemical quantities involved in the process. For the case of a turbulent flame one needs to define a PDF to compute the Favre average of the thermochemical quantities. In particular, in the present work, the authors are interested in the modeling of the PDF function required to evaluate chemical Favre-averaged quantities. The definition of such a function is critical due to the poor knowledge of the behavior of two independent variables. The aim of this work is to provide an extension of the standard FPV model for turbulent combustion, applying the SMLD [6] approach to the progress variable $\mathrm{PDF}$, maintaining a good compromise between computational costs and accuracy level. 
In section 2, the rational for the definition of such a PDF is presented. Then, three PDF models are considered and their role in the evaluation of nonpremixed flames is analyzed. In section 3, the numerical results obtained in the simulation of the Sandia flames [7] and of supersonic combustion [8] are discussed. Summary and conclusions close the paper.

\section{COMBUSTION MODEL}

\subsection{The Flamelet Approach}

In this work, the FPV model proposed by Pierce [2] is used to evaluate all of the thermochemical quantities involved in the combustion process. This approach is based on the parametrization of the generic thermochemical quantities, $\phi$, in terms of two variables: the mixture fraction $Z$ and the progress variable $C$ :

$$
\phi=F_{\phi}(Z, C) \text {. }
$$

Equation (1) is taken as the solution of the steady laminar flamelet equation:

$$
-\rho \frac{\chi}{2} \frac{\partial^{2} \phi}{\partial Z^{2}}=\dot{\omega}_{\phi}
$$

where $\chi$ is the scalar dissipation rate modeled in terms of molecular diffusivity of mixture fraction $D_{Z}, \chi=2 D_{Z}(\nabla Z)^{2} ; \rho$ is the density; and $\dot{\omega}_{\phi}$ is the source term related to $\phi[2]$. Each solution of Eq. (2), is a flamelet and the solution variety over $\chi=\chi_{\text {st }}$, called S-curve, is shown in Fig. 1. From Eq. (1), one can obtain the Favre averages of $\phi$ using the definitions:

$$
\begin{aligned}
\widetilde{\phi} & =\iint F_{\phi}(Z, C) \widetilde{P}(Z, C) d Z d C ; \\
\widetilde{\phi^{\prime \prime 2}} & =\iint\left(F_{\phi}(Z, C)-\widetilde{\phi}\right)^{2} \widetilde{P}(Z, C) d Z d C .
\end{aligned}
$$

Here, $\widetilde{P}(Z, C)$ is the density-weighted PDF:

$$
\widetilde{P}(Z, C)=\frac{\rho P(Z, C)}{\bar{\rho}}
$$

where $P(Z, C)$ is the PDF and $\bar{\rho}$ is the Reynolds-averaged density. As usual, $\phi$ is decomposed as

$$
\phi=\widetilde{\phi}+\phi^{\prime \prime} ; \quad \widetilde{\phi}=\frac{\overline{\rho \phi}}{\bar{\rho}}
$$




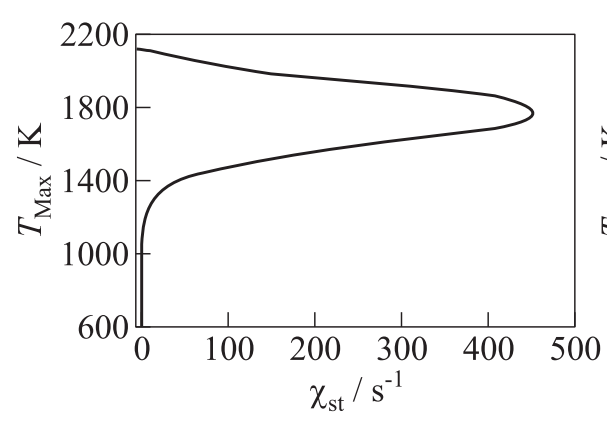

(a)

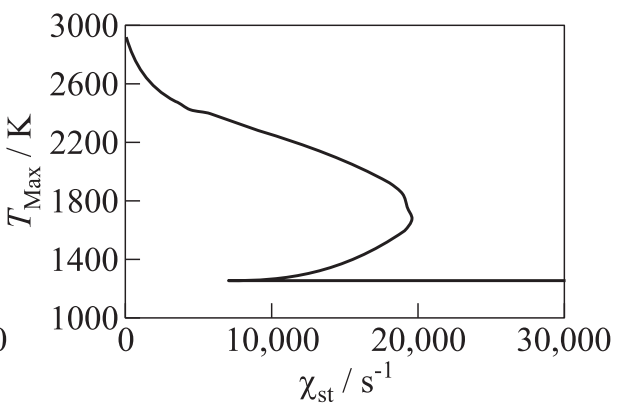

(b)

Figure 1 S-shaped curve in the plot of the maximum temperature of each flamelet vs. the scalar stoichiometric dissipation rate: $(a) \mathrm{H}_{2}$-air counterflow combustion at ambient pressure and hydrogen and air temperatures of 545 and $1250 \mathrm{~K}$, respectively; and $(b) \mathrm{CH}_{4}$-air counterflow combustion at ambient pressure and methane and air temperatures of 294 and $291 \mathrm{~K}$, respectively

and

$$
\rho=\bar{\rho}+\rho^{\prime}
$$

with $\phi^{\prime \prime}$ and $\rho^{\prime}$ being the fluctuations.

The choice of such a PDF plays a crucial role in the definition of the model being a compromise between computational costs and accuracy level. In this respect, this paper provides an extension of the standard FPV turbulent combustion model combined with a Reynolds-averaged Navier-Stokes (RANS) equation solver [9] where different fundamental hypotheses are used to define the $\mathrm{PDF}$ function for the progress variable, $C$. The influence of different PDFs in the simulation of nonpremixed turbulent combustion is the final aim of this research.

\subsection{Presumed Probability Density Function Modeling}

In order to investigate the role of the presumed PDF, one can, first of all, use the Bayes' theorem and take the PDF as the product between the marginal PDF of $Z$ and the conditional $\mathrm{PDF}$ of $C \mid Z$ :

$$
\widetilde{P}(Z, C)=\widetilde{P}(Z) P(C \mid Z) .
$$

Therefore, one has to presume, or evaluate, the functional shape of such PDFs. Let us consider the marginal $\mathrm{PDF}, \widetilde{P}(Z)$. By several authors, it has been shown that the mixture fraction is best described by a passive scalar and that the $\mathrm{PDF}$ of a passive scalar can be approximated by a $\beta$ distribution function $[10$ 
12]. The two-parameter family of $\beta$-distribution in the interval $x \in[0,1]$ is given by

$$
P(x)=x^{a-1}(1-x)^{b-1} \frac{\Gamma(a+b)}{\Gamma(a) \Gamma(b)}
$$

where $\Gamma(x)$ is the Euler function and $a$ and $b$ are two parameters related to $\widetilde{x}$ and $\widetilde{x^{\prime \prime 2}}$, respectively:

$$
a=\frac{\widetilde{x}\left(\widetilde{x}-\widetilde{x}^{2}-\widetilde{x^{\prime \prime 2}}\right)}{\widetilde{x^{\prime \prime 2}}} ; \quad b=\frac{(1-\widetilde{x})\left(\widetilde{x}-\widetilde{x}^{2}-\widetilde{x^{\prime \prime 2}}\right)}{\widetilde{x^{\prime \prime 2}}} .
$$

For all the three models presented here, the $\beta$-distribution is employed for $\widetilde{P}(Z)$.

To presume the functional shape of the distribution of a reacting scalar, one needs to make some constitutive hypotheses. In this work, to simplify the problem, the statistical independence of $Z$ and $C$ is assumed so that for all of the considered models, $\widetilde{P}(Z, C)=\widetilde{P}(Z) P(C)$, namely, $C=C \mid Z$. The most widely used hypothesis (model A), implying great simplification in the theoretical framework, consists in assuming that the conditional PDF, $P(C)$, can be modeled by a Dirac distribution. It can be shown that there is only one solution of Eq. (2) for each chemical state. With this criterion, the Favre-average of a generic thermochemical quantity is given by:

$$
\widetilde{\phi}=\iint F_{\phi}(Z, C) \widetilde{\beta}(Z) \delta(C-\widetilde{C}) d Z d C=\int F_{\phi}(Z, \widetilde{C}) \widetilde{\beta}(Z) d Z .
$$

Therefore, one has only three additional transport equations (for $\widetilde{Z}, \widetilde{Z^{\prime \prime 2}}$, and $\widetilde{C}$ ) to evaluate all thermochemical quantities in the flow, thus avoiding the expensive solution of a transport equation for each chemical species. However, it is well known that a reactive scalar [13], such as $C$, depends on a combination of solutions of Eq. (2) for each chemical state and, therefore, its PDF cannot be accurately approximated by a Dirac distribution.

Therefore, the second model (model $\mathrm{B}$ ) is given by assuming that $Z$ and $C$ are distributed in the same way, namely, using a $\beta$-distribution, thus giving the following joint PDF:

$$
\widetilde{P}(Z, C)=\widetilde{\beta}(Z) \beta(C) .
$$

This avoids the simplification seen before and, consequently, the model requires the evaluation of an additional transport equation for $\widetilde{C^{\prime \prime 2}}$.

Moreover, the probability distribution of a reacting scalar is often multimodal, unlike the $\beta$ function, and its functional form depends on the turbulencechemistry interaction. Therefore, one can think about a distribution built considering, as constraints, the only available information, namely, on the values of $\widetilde{Z}, \widetilde{Z^{\prime \prime 2}}, \widetilde{C}$, and $\widetilde{C^{\prime \prime 2}}$. 
The third model (model $\mathrm{C}$ ) is obtained evaluating the conditional PDF as the SMLD [13]. It can be shown that if one knows only its first three moments, the PDF can be evaluated using "Laplace's principle of insufficient reason" [6]. The technique is developed following the statistical mechanics arguments presented by Heinz [14]. Relying on the knowledge of the first three moments of $P(C)$, a unique measure, $S$, of the predictability of a thermodynamic state can be defined, where $S$ is the entropy function depending on $P(C), S=S(P(C))[15]$ that can be thought of as the Boltzmann's entropy:

$$
S=-\int P(C) \ln \left(\frac{P(C)}{Q(C)}\right) d C
$$

where $Q(C)$ is the bias density function to integrate information when no moments are known. In this paper, the form of $Q(C)$ proposed by Pope, discussed in [13], is assumed. The goal is to construct a PDF that maximizes the entropy $S$. Following the Lagrangian optimization approach, the functional $S^{*}$ is defined by involving the constraints on the moments:

$$
S^{*}=-\int d C\left\{P(C) \ln \left(\frac{P(C)}{Q(C)}\right)+\sum_{n=1}^{2} \mu_{n} C^{n} P(C)-\frac{P(C)}{Q(C)}\right\} .
$$

In the above equation, $\mu_{n}$ are the Lagrange's multipliers while the last fraction term is introduced to normalize $P(C)$. The expression for $P(C)$, obtained evaluating the maximum of $S^{*}$, reads:

$$
P(C)=\frac{1}{\mu_{0}} \exp \left\{-\sum_{n=1}^{2} \frac{\mu_{n}}{n}(C-\widetilde{C})^{n}\right\}
$$

where

$$
\begin{aligned}
\mu_{0} & =\int_{0}^{1} d C P(C) \\
-\mu_{1} & =\int_{0}^{1} d C \partial_{C}(P(C))=P(1)-P(0) ; \\
1-\mu_{2} \widetilde{C^{\prime \prime 2}} & =\int_{0}^{1} d C \partial_{C}[(C-\widetilde{C}) P(C)]=P(1)-C \mu_{1}
\end{aligned}
$$

since $Z$ and $C$ are bounded in $[0,1]$.

At this point, the model still needs an additional assumption to be closed. Here, differently from [13], let assume that the first and the last points of $P(C)$ 
are equal to the first and last points of $\beta(C)$ evaluated with the given values of the mean and variance:

$$
P\left(1 ; \widetilde{C}, \widetilde{C^{\prime \prime 2}}\right)=\beta\left(1 ; \widetilde{C}, \widetilde{C^{\prime \prime 2}}\right) ; \quad P\left(0 ; \widetilde{C}, \widetilde{C^{\prime \prime 2}}\right)=\beta\left(0 ; \widetilde{C}, \widetilde{C^{\prime \prime 2}}\right) .
$$

This assumption does not affect the multimodal nature of the distribution, but simplifies the model implementation (there is no need to evaluate the roots of a nonlinear system) with respect to the original scheme [13]. The major advantage of the SMLD approach over conventionally employed presumed PDF closure models is that it provides a systematic framework to incorporate an arbitrary number of moment information. It is noteworthy that, since $C$ is used instead of $C \mid Z$ as argument of $P$, also this model assumes statistical independence of $Z$ and $C$.

For the case of turbulent flames, the solution of the steady-laminar flamelet equation, namely, Eq. (1), is expressed in terms of the Favre averages of $Z$ and $C$ and of their variance. Using model $\mathrm{A}$, one can tabulate all chemical quantities in terms of $\widetilde{Z}, \widetilde{Z^{\prime \prime 2}}$, and $\widetilde{C}$, since, due to the properties of the $\delta$-distribution, the model is independent of $\widetilde{C^{\prime \prime 2}}$. On the other hand, models $\mathrm{B}$ and $\mathrm{C}$ express $\phi$ also in terms of $\widetilde{C^{\prime \prime 2}}$ and, therefore, an additional transport equation needs to be solved. In this case, the transport equations for the combustion model reads:

$$
\begin{aligned}
& \partial_{t}(\bar{\rho} \widetilde{Z})+\vec{\nabla} \cdot(\bar{\rho} \widetilde{\vec{u}} \widetilde{Z})=\vec{\nabla} \cdot\left[\left(D+D_{\widetilde{Z}}^{t}\right) \bar{\rho} \vec{\nabla} \widetilde{Z}\right] ; \\
& \partial_{t}\left(\bar{\rho} \widetilde{Z^{\prime \prime 2}}\right)+\vec{\nabla} \cdot\left(\bar{\rho} \widetilde{\vec{u}} \widetilde{Z^{\prime \prime 2}}\right)=\vec{\nabla} \cdot\left[\left(D+D_{\widetilde{Z^{\prime \prime 2}}}^{t}\right) \bar{\rho} \vec{\nabla} \widetilde{Z^{\prime \prime 2}}\right]-\bar{\rho} \widetilde{\chi}+2 \bar{\rho} D_{\widetilde{Z}}^{t}(\vec{\nabla} \widetilde{Z})^{2} ; \\
& \partial_{t}(\bar{\rho} \widetilde{C})+\vec{\nabla} \cdot(\bar{\rho} \widetilde{\vec{u}} \widetilde{C})=\vec{\nabla} \cdot\left[\left(D+D_{\widetilde{C}}^{t}\right) \bar{\rho} \vec{\nabla} \widetilde{C}\right]+\bar{\rho} \overline{\dot{\omega}_{C}} \\
& \partial_{t}\left(\bar{\rho} \widetilde{C^{\prime \prime 2}}\right)+\vec{\nabla} \cdot\left(\bar{\rho} \widetilde{\vec{u}} \widetilde{C^{\prime \prime 2}}\right)=\vec{\nabla} \cdot\left[\left(D+D_{\widetilde{C^{\prime \prime 2}}}^{t}\right) \bar{\rho} \vec{\nabla} \widetilde{C^{\prime \prime 2}}\right]-\bar{\rho} \widetilde{\chi} C \\
&+2 \bar{\rho} D_{\widetilde{C}}^{t}(\vec{\nabla} \widetilde{C})^{2}+2 \bar{\rho} \widetilde{C^{\prime \prime} \dot{\omega}_{C}^{\prime \prime}}
\end{aligned}
$$

where $\widetilde{\chi_{C}}$ is modeled in terms of $\widetilde{Z^{\prime \prime 2}}$ and $\widetilde{C^{\prime \prime 2}}[16]$, namely, $\widetilde{\chi_{C}}=\widetilde{Z^{\prime \prime 2}} \chi / \widetilde{C^{\prime \prime 2}}$; $D$ is the diffusion coefficient for all of the species, given as $D=\nu / \operatorname{Pr}$ assuming a unity Lewis number; $\nu$ and $\operatorname{Pr}$ are the kinematic viscosity and the Prandtl number, respectively; $D_{\widetilde{Z}}^{t}=D_{\widetilde{Z^{\prime \prime 2}}}^{t}=D_{\widetilde{C}}^{t}=D_{\widetilde{C^{\prime \prime 2}}}^{t}=\nu_{t} / \mathrm{Sc}_{t}$ are the turbulent mass diffusion coefficients, $\mathrm{Sc}_{t}$ being the turbulent Schmidt number; and, finally, $\dot{\omega}_{C}$ is the source term for the progress variable precomputed and tabulated in the flamelet library. The gradient transport assumption for turbulent fluxes is used and the mean scalar dissipation rate, $\widetilde{\chi}$ and $\tilde{\chi}_{C}$, appear as sink terms in Eqs. (5) and (7), respectively.

At each iteration, the values of the flamelet variables are updated using Eqs. (4)-(7) and the Favre-averaged thermochemical quantities are computed, 
using Eq. (3). Such solutions provide the mean mass fractions which are used to evaluate all of the transport properties of the fluid, namely, the molecular viscosity, the thermal conductivity, and the species diffusion coefficients.

\section{FLOW EQUATIONS AND NUMERICAL SOLUTION}

The numerical method developed in [9] has been employed to solve the steadystate RANS equations with $k-\omega$ turbulence closure. For an axisymmetric multicomponent reacting compressible flow, the system of the governing equations can be written as

$$
\partial_{t} \vec{Q}+\partial_{x}\left(\vec{E}-\vec{E}_{\nu}\right)+\partial_{y}\left(\vec{F}-\vec{F}_{\nu}\right)=\vec{S} .
$$

Here, $t$ is the time variable; $x$ and $y$ are the axial and the radial coordinates, respectively; $\vec{Q}$ is the vector of the conserved variables

$$
\vec{Q}=\left(\bar{\rho}, \bar{\rho} \widetilde{u}_{x}, \bar{\rho} \widetilde{u}_{y}, \bar{\rho} \widetilde{H}-p_{t}, \bar{\rho} k, \bar{\rho} \omega, \bar{\rho} \widetilde{R}_{n}\right)
$$

where $k$ and $\omega$ are the turbulence kinetic energy and its specific dissipation rate, $\bar{\rho},\left(\widetilde{u}_{x}, \widetilde{u}_{y}\right)$, and $\widetilde{H}$ indicates the Reynolds-averaged value of density, the Favreaveraged values of velocity components, and the specific total enthalpy given by

$$
\widetilde{H}=\widetilde{h}+\frac{1}{2}\left(\widetilde{u}_{x}^{2}+\widetilde{u}_{y}^{2}\right)+\frac{5}{3} k
$$

with $\widetilde{h}$ accounting for the species enthalpy per unit mass, respectively; $p_{t}$ is given by the state equation, namely, $p_{t}=\bar{\rho} \widetilde{R} \widetilde{T}$, with $\widetilde{R}$ and $\widetilde{T}$ being the Favreaveraged values of the gas constant and of the temperature, respectively; and $\widetilde{R}_{n}$ is the generic set of conserved variables related to the combustion model. In this framework, $\widetilde{R}_{n}$ is the set of independent variables of the flamelet model, namely, $\widetilde{Z}, \widetilde{Z^{\prime \prime 2}}, \widetilde{C}$, and $\widetilde{C^{\prime \prime 2}}$ (see the previous subsection); $\vec{E}, \vec{F}$ and $\vec{E}_{v}, \vec{F}_{v}$ are the inviscid and viscous flux vectors [17], respectively; and $\vec{S}$ is the vector of the source terms.

The heat flux in the total energy equation, namely,

$$
q=-\rho c_{p} D_{T} \nabla T+\sum_{n=1}^{N_{s}} \rho V_{n} Y_{n} h_{n},
$$

is composed of two terms since the Dufour effect is neglected, $D_{T}$ is the thermal diffusivity and $c_{p}$ is the specific heat at constant pressure. The mass diffusion term is treated with the Fick's law considering

$$
V_{n}=-\frac{D_{n, \operatorname{mix}} \nabla Y_{n}}{Y_{n}}
$$

assigning a mixture diffusivity, $D_{n, \mathrm{mix}}$, to each species. 
A cell-centered finite volume space discretization is used on a multiblock structured mesh. The convective and viscous terms are discretized by the thirdorder-accurate Steger and Warming [18] flux-vector-splitting scheme and by second-order-accurate central differences, respectively. An implicit time marching procedure is used with a factorization based on the diagonalization procedure of Pulliamm and Chaussee [19], employing a scalar alternating direction implicit (ADI) solution procedure [20]. Steady flows are considered and the ADI scheme is iterated in the pseudotime until a residual drop of at least five orders of magnitude for all of the conservation-law Eq. (8) is achieved. Characteristic boundary conditions for the flow variables are imposed at inflow and outflow points, whereas no slip and adiabatic conditions are imposed at walls; $k, \omega$, and $\tilde{R}_{n}$ are assigned at inflow points, whereas they are linearly extrapolated at outflow points. At solid walls, $k$ is set to zero and $\omega$ is evaluated as proposed in $[21]$ :

$$
\omega=60 \frac{\nu}{0.09 y_{n, 1}^{2}}
$$

where $y_{n, 1}$ is the distance of the first cell center from the wall; the homogeneous Neumann boundary condition is used for $\tilde{R}_{n}$ (noncatalytic wall). Finally, symmetry conditions are imposed at the axis.

\section{NUMERICAL RESULTS}

This section provides the comparison among the results obtained using the three combustion models so as to assess the influence that the PDF choice may have in the prediction of turbulent nonpremixed flames.

The well-known subsonic Sandia flames [7] are considered at first; then, a supersonic test case is analyzed, whose experimental data are available in the literature [8]. The flamelet library has been computed over a grid with 250 uniformly distributed points, in the $\widetilde{Z}$ and $\widetilde{C}$ directions, and 50 uniformly distributed points in the $\widetilde{Z^{\prime \prime 2}}$ and $\widetilde{C^{\prime \prime 2}}$ directions and a quadri-linear interpolation scheme is used. Indeed, when considering model A, the grid is built considering only $\widetilde{Z}, \widetilde{Z^{\prime \prime 2}}$, and $\widetilde{C}$, with the same spacing. The boundary conditions adopted to evaluate the flamelet library are exactly the air stream and the fuel jet conditions for the two cases but for the pressure that is almost constant in both cases and assumed as the atmospheric one in order to recover the boundary conditions of the mixture fraction and the progress variable. Indeed, the air stream is represented as $\widetilde{Z}=0$ and $\widetilde{C}=0$, while the fuel jet is given by $\widetilde{Z}=1$ and $\widetilde{C}=0$. The steady flamelet evaluations, for both test cases, have been performed using the FlameMaster code [22]. 


\subsection{Sandia Flames Test Case}

The Sandia Flames presented here are two different piloted partially premixed methane-air diffusion flames burning at the same pressure equal to $100.6 \mathrm{kPa}$ and at two different Reynolds numbers, Re, based on the nozzle diameter, the jet bulk velocity, and the kinematic viscosity of the fuel. The diameter of the nozzle of the central jet is $d_{\text {ref }}=7.2 \mathrm{~mm}$ and the internal and external diameters of the annular pilot nozzle are equal to 7.7 and $18.2 \mathrm{~mm}$, respectively. The

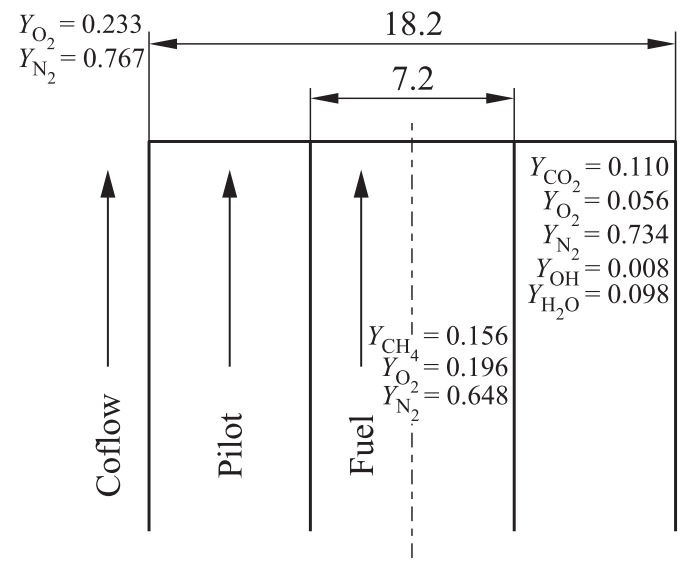

(a)
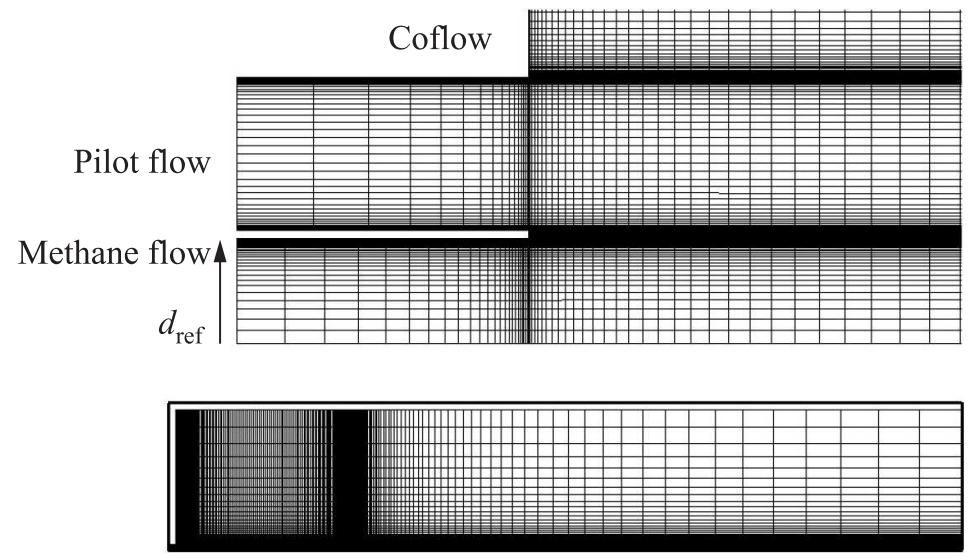

(b)

Figure 2 Schematic of the Sandia Flame burner $(a)$ and flow field discretization $(b)$. Dimensions are in millimeters 
fluid jet is a mixture of $75 \%$ air and $25 \%$ methane by volume. Partial premixing with air reduces the flame length and produces more stable flames than pure $\mathrm{CH}_{4}$ [7]. Consequently, the flame may be operated at high Re with little effect of local extinction even with little pilot. Flame D $(\mathrm{Re}=22,400)$ presents a very low degree of local extinction, whereas Flame $\mathrm{E}(\mathrm{Re}=33,600)$ has a significant and increasing probability of local extinction near the pilot. The pilot is a mixture of air with the main methane combustion products, namely, $\mathrm{C}_{2} \mathrm{H}_{2}$, $\mathrm{H}_{2}, \mathrm{CO}_{2}$, and $\mathrm{N}_{2}$, with the same enthalpy at the equivalence ratio $\Phi=0.77$ corresponding to the equilibrium composition $\widetilde{Z}=0.27, \widetilde{Z^{\prime \prime 2}}=0.0075, \widetilde{C}=1$, and $\widehat{C^{\prime \prime 2}}=0$. The oxidizer air $\left(Y_{\mathrm{O}_{2}}=0.233\right.$ and $\left.Y_{\mathrm{N}_{2}}=0.767\right)$ is supplied as a coflow at $291 \mathrm{~K}$. The schematic of the Sandia Flame burner is shown in Fig. 2.

The computational domain is axisymmetric and includes a part of the burner; it has a length of $150 d_{\text {ref }}$ and $27 d_{\text {ref }}$ along the axial and radial directions, respectively, and has been discretized using about 45,000 cells; Fig. $2 b$ shows the discretization used with a closeup of the near-burner region. The reference length, $d_{\text {ref }}$, on the burner is discretized with 40 geometrically distributed points in the radial direction. The smallest cell is given by a rectangular element of $10^{-5} \times 0.5 \cdot 10^{-5} \mathrm{~m}$. Computations have been carried out using the com-
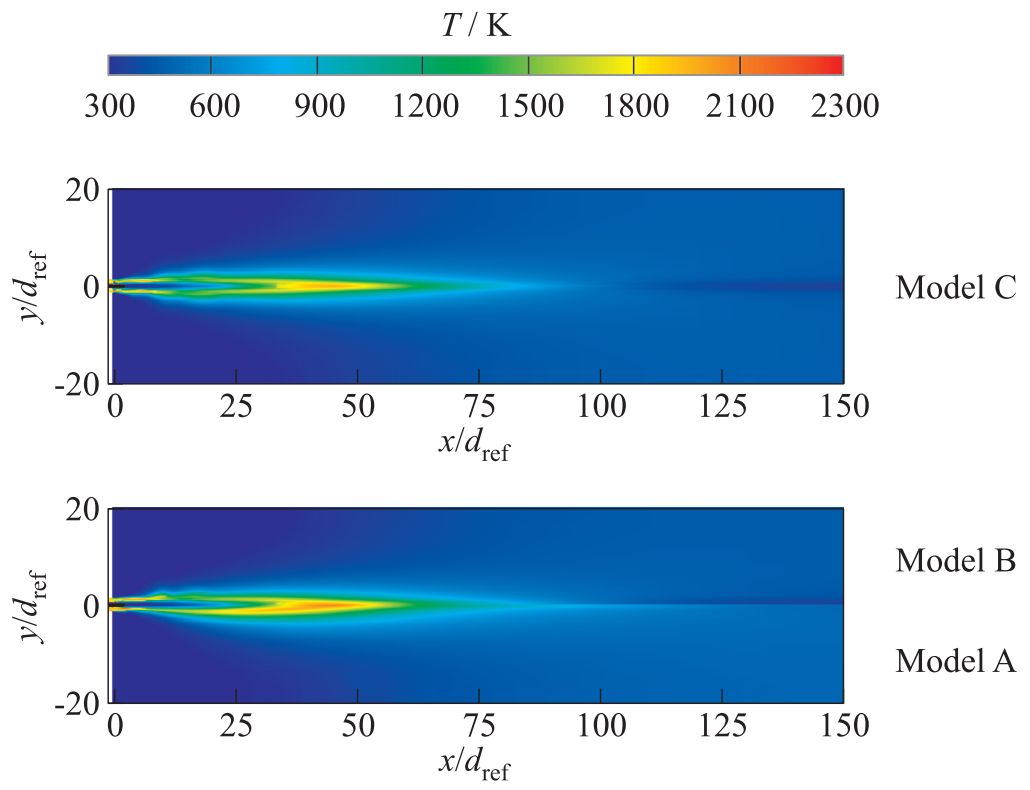

Figure 3 Temperature contours for the Sandia Flame E 

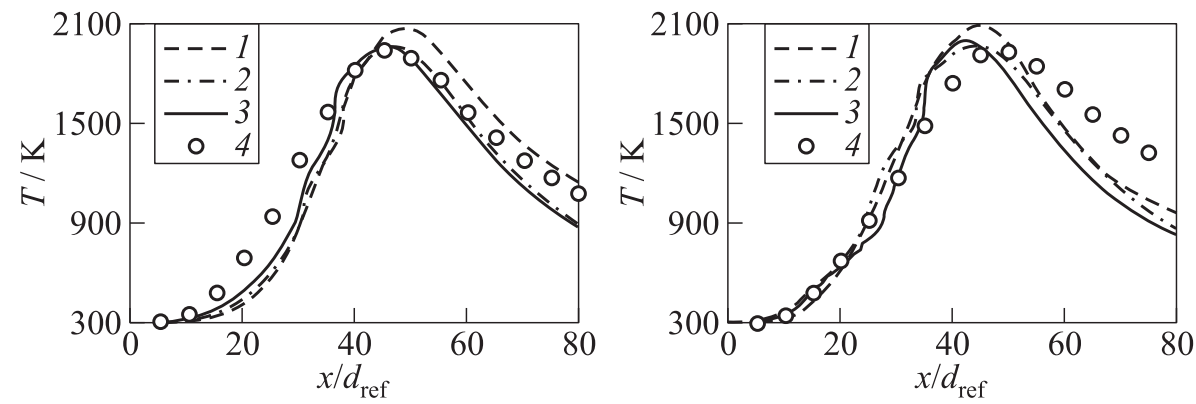

(a)
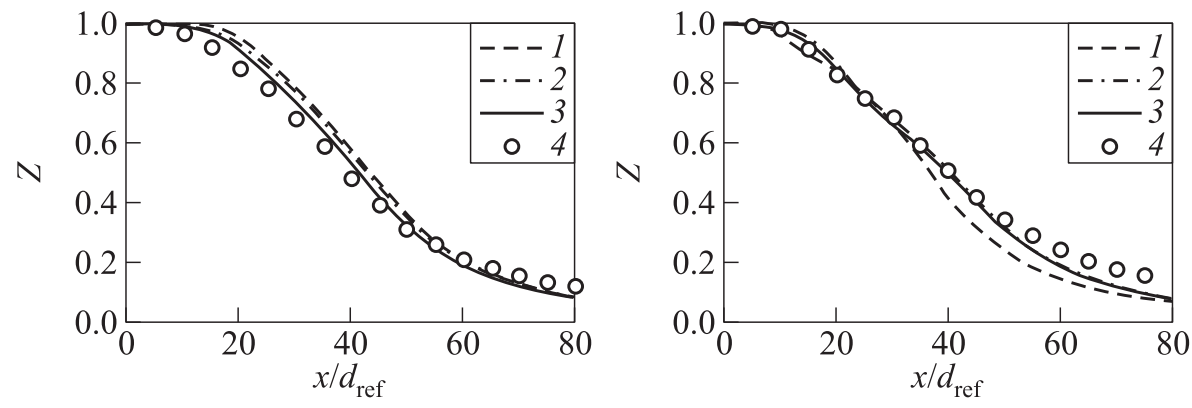

(b)

Figure 4 Temperature $(a)$ and mixture fraction $(b)$ distributions along axis of Flame D (left column) and Flame E (right column): 1 - model A; 2 - model B; 3 - model $\mathrm{C}$; and 4 - experimental data

bustion scheme described by the GRI-MECH 3.0 [23]: 325 subreactions upon 53 species.

The temperature field obtained by the three combustion models for the case of the Sandia Flame E is presented in Fig. 3. Moreover, Fig. 4 provides the temperature distributions along the axial direction. It appears that in the nearburner region, models $\mathrm{B}$ and $\mathrm{C}$ show a better agreement with the experimental data than model A. Moving away from the burner $\left(x>20 d_{\text {ref }}\right)$, the agreement deteriorates; this is probably due to the accuracy limits of the RANS approach in the prediction of the mixing process that greatly affects combustion. From these two sets of figures, one can see that there is a slight improvement provided by model $\mathrm{C}$ in the evaluation of the flame core and of the flame shapes.

The radial distributions of temperature, mixture fraction, mixture fraction variance, and $\mathrm{H}_{2} \mathrm{O}$ mass fraction at several sections taken at the axial coordinate $x / d_{\text {ref }}$ equal to 1,15 , and 30 are shown in Figs. 5 and 6 for Flame D and 

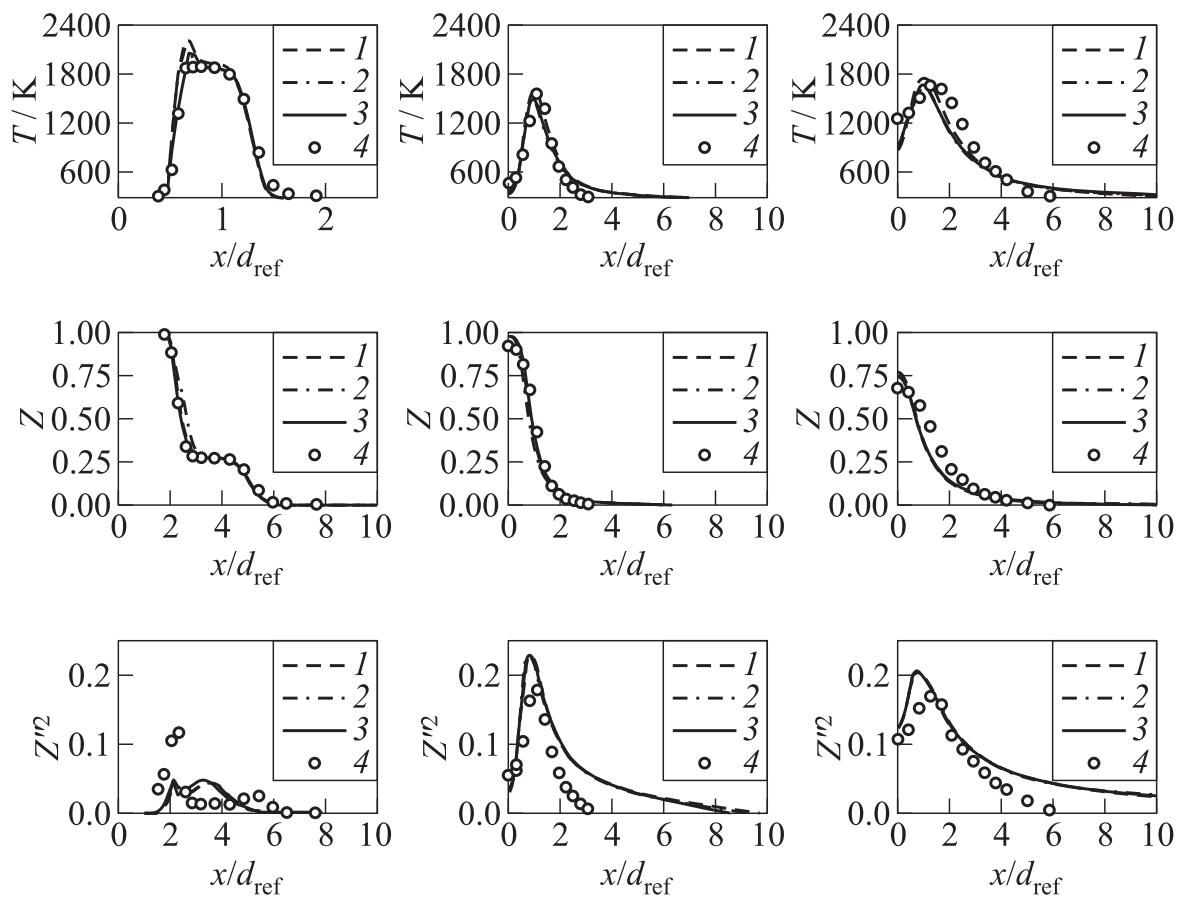

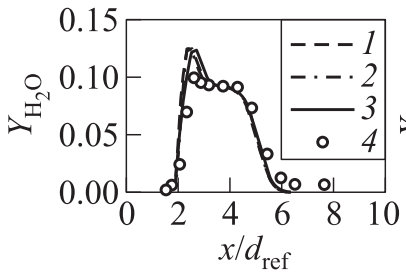

(a)

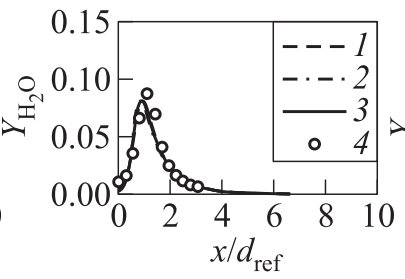

(b)

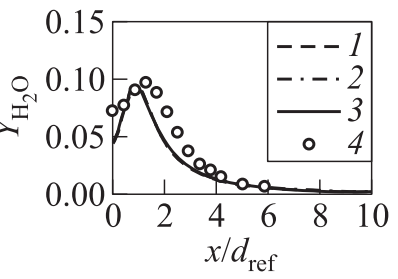

(c)

Figure 5 Thermodynamic quantities distributions in Sandia Flame D along several sections taken at $x / d_{\text {ref }}=1(a), 15(b)$, and $30(c)$ from the burner: 1 - model $\mathrm{A}$; 2 - model B; 3 - model $\mathrm{C}$; and 4 - experimental data

Flame E, respectively. One can see that the results of model B (corresponding to assumption that $\widetilde{P}(Z, C)=\widetilde{\beta}(Z) \beta(C)$ ) and model $\mathrm{C}$ (corresponding to the choice $\left.\widetilde{P}(Z, C)=\widetilde{\beta}(Z) P_{\mathrm{SML}, 2}(C)\right)$ are in better agreement with the experimental data [7] than the results of model A (corresponding to the standard choice $\widetilde{P}(Z, C)=\widetilde{\beta}(Z) \delta(C-\widetilde{C \mid Z}))$. Envisaging a model that does not require the hypothesis of statistically independence of $Z$ and $C$ should considerably enhance the prediction accuracy also in the regions close to the burner. 

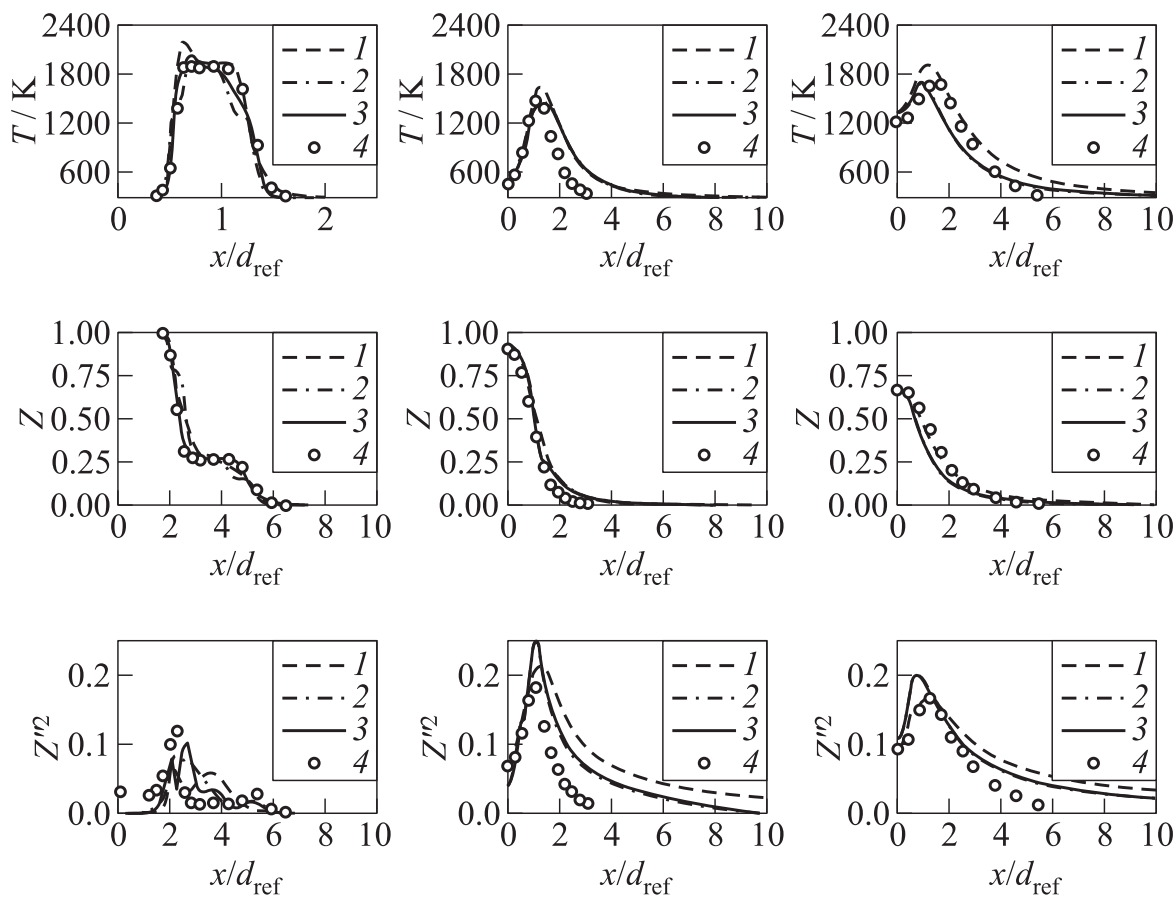

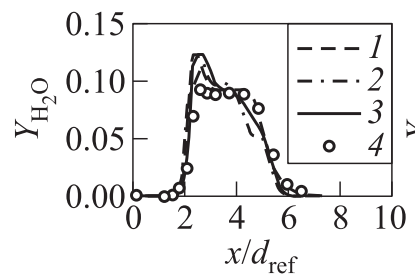

(a)

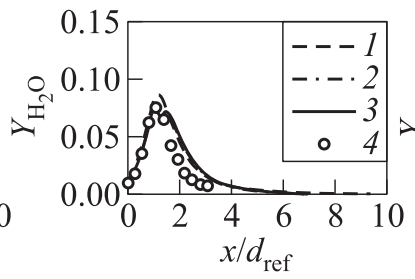

(b)

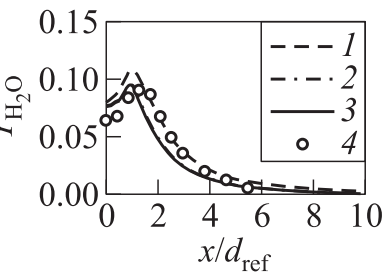

(c)

Figure 6 Thermodynamic quantities distributions in Sandia Flame E along several sections taken at $x / d_{\mathrm{ref}}=1(a), 15(b)$, and $30(c)$ from the burner: 1 - model A; 2 - model B; 3 - model $\mathrm{C}$; and 4 - experimental data

\subsection{Supersonic Combustion Test Case}

The test case presented is the supersonic hydrogen-air combustor at the NASA Langley Research Center proposed by Cheng et al. [8].

Figure $7 a$ shows the supersonic burner that provides an annular axisymmetric jet of hot vitiated wet air at Mach number equal to 2, average axial velocity of $1420 \mathrm{~m} / \mathrm{s}$, temperature of $1250 \mathrm{~K}$, and pressure of $107 \mathrm{kPa}$. The composition 


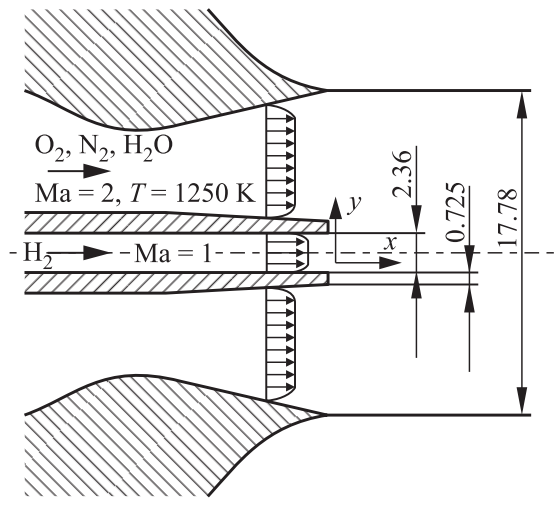

(a)
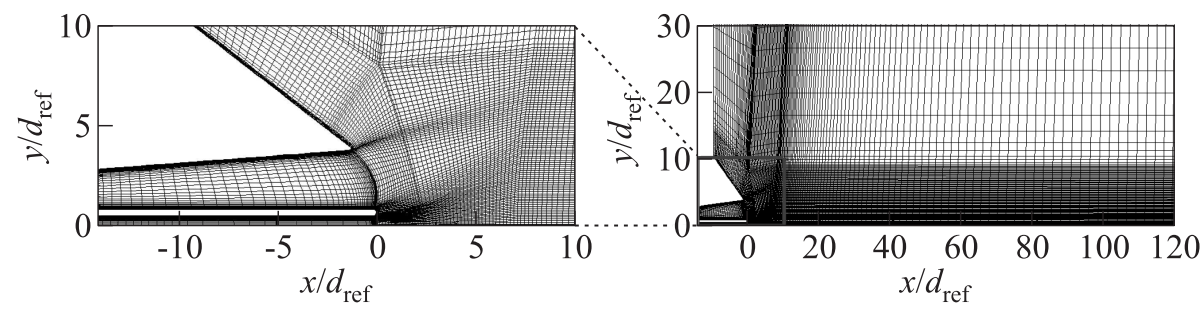

(b)

Figure 7 Schematic of the Cheng's flame burner [8] (dimensions are in millimeters) (a) and flow field discretization $(b)$

of the vitiated air is: $Y_{\mathrm{O}_{2}}=0.201, Y_{\mathrm{H}_{2} \mathrm{O}}=0.255$ and $Y_{\mathrm{N}_{2}}=0.544$. The exit conditions of the air stream are given by a precombustion at low temperature [8]. The hydrogen exit is estimated as a chocked flow with average axial velocity of $1780 \mathrm{~m} / \mathrm{s}$, temperature of $545 \mathrm{~K}$, and pressure of $112 \mathrm{kPa}$. The diameter of the fuel stream is $d_{\text {ref }}=2.362 \mathrm{~mm}$, taken as the reference length. At the inlet section, the inner diameter of the vitiated air stream is $3.812 \mathrm{~mm}$ and its outer diameter is $17.78 \mathrm{~mm}$ [25], respectively.

The computational domain is axisymmetric and includes the divergent part of the air nozzle; it extends $150 d_{\text {ref }}$ and $50 d_{\text {ref }}$ along the axial and radial directions, respectively, and has been discretized using about 100,000 cells; Fig. $7 b$ shows the discretization used. The reference length, $d_{\text {ref }}$, on the burner is discretized with 100 geometrically distributed points in the radial direction. The smallest cell is given by a rectangular element of $0.2 \cdot 10^{-5} \times 0.1 \cdot 10^{-5} \mathrm{~m}$. The evaluation of the flamelet library has been performed using the Vajda et al. kinetic scheme [26]: 19 subreactions upon 7 species. In view of the results of the 


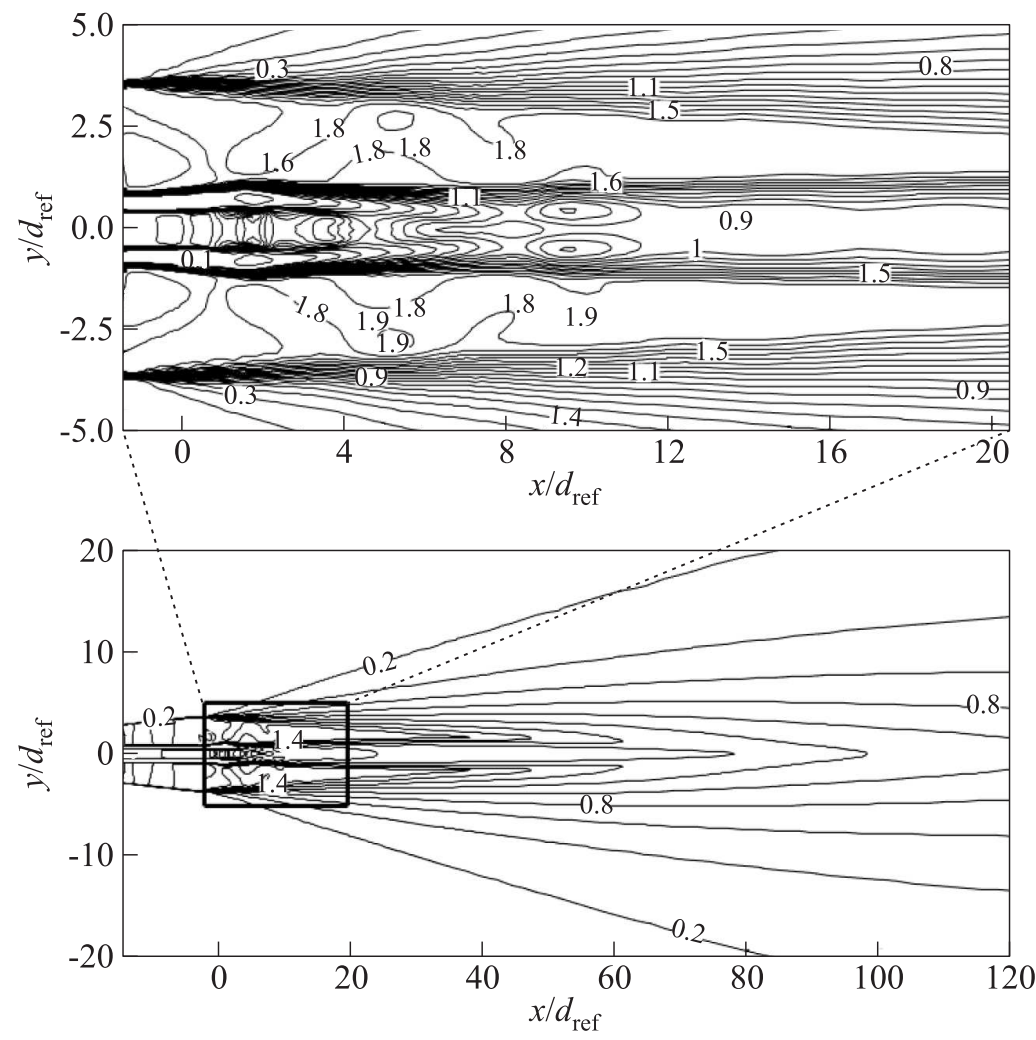

Figure 8 Mach's number isolines with a closeup of the near-burner region of the Cheng's Flame combustion chamber

previous section, in the following only the results of models $\mathrm{A}$ and $\mathrm{C}$ will be shown and discussed.

Figure 8 shows the Mach number contours in the flow obtained using model C. Through the closeup of the near-burner region, it is shown that the initial velocity condition are fulfilled.

Figure 9 presents a qualitative comparison between the temperature distributions evaluated with models $\mathrm{A}$ and $\mathrm{C}$, showing the slightly different predictions of the jet shape. Model $\mathrm{A}$ evaluates a reaction zone attached to the burner that is longer than the one evaluated with model C: the first one extends up to about $4 d_{\text {ref }}$ while the second one extends up to about $2 d_{\text {ref. }}$ The maximum temperature predicted by using model $\mathrm{A}$ is higher than that obtained by model $\mathrm{C}$ : 2800 vs. $2600 \mathrm{~K}$. The high temperature region is found near the axis, at a distance in the range $(30-60) d_{\text {ref }}$ from the burner. On the other hand, model $\mathrm{C}$ 


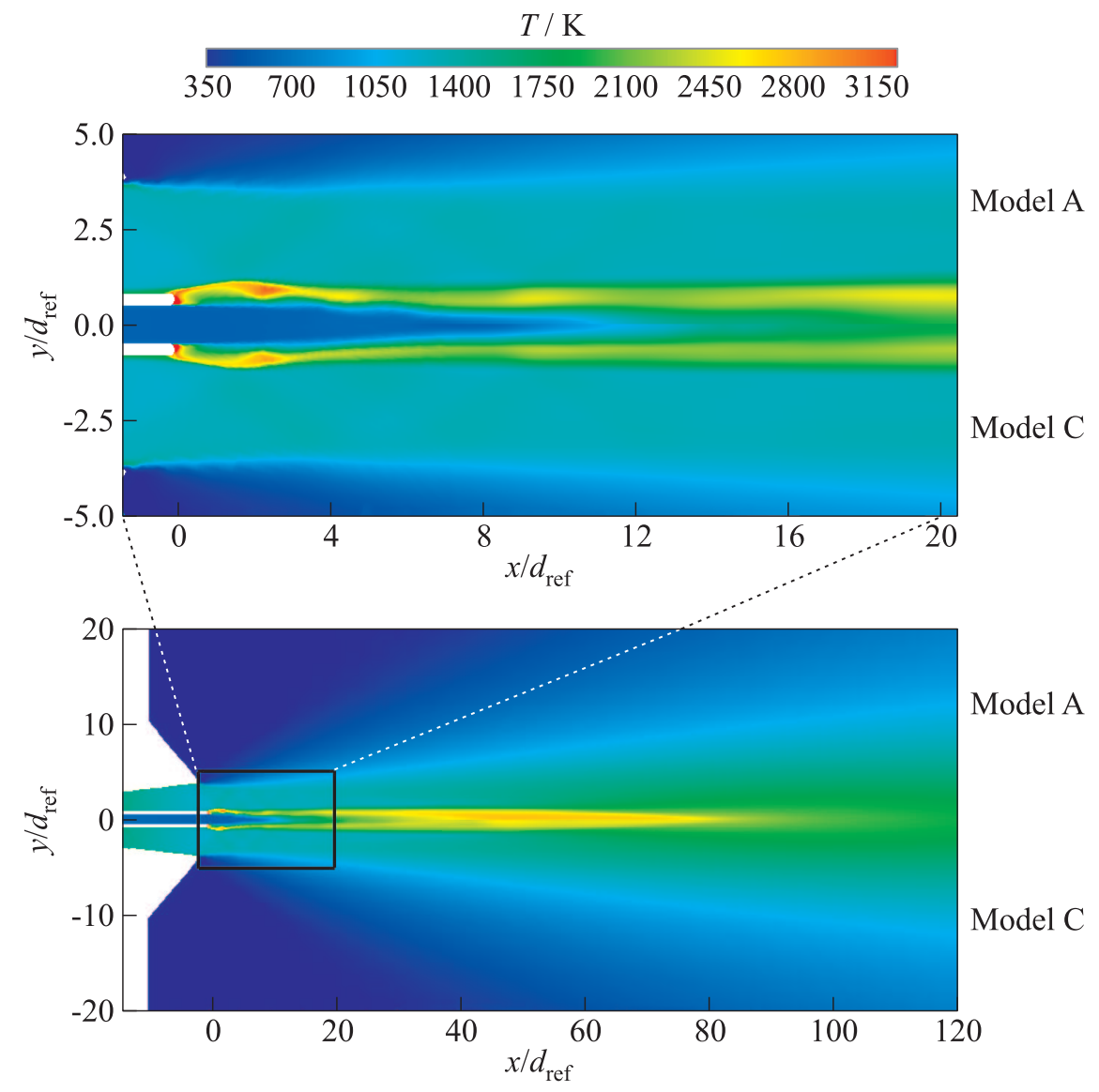

Figure 9 Temperature distribution of the Cheng's flame evaluated with the two models

still predicts an attached flame, the improvement with respect to model A appearing marginal. This is due to the fact that FPV models are built in the low Mach number assumption [27] so that both models $\mathrm{A}$ and $\mathrm{C}$ estimate that the reactions may occur in the entire region in which the two flows $\left(\mathrm{H}_{2}-\right.$ air $)$ come up against each other, showing an absolutely fast reaction rate for the combustion process. In this context, a correct evaluation of the statistical correlation between $Z$ and $C$ should turn in an improved accuracy since the kinetic should be better modeled. Considering the proper statistical behavior of the progress variable with respect to the mixture fraction variations, namely, $P(C \mid Z)$ and not $P(C)$, one should be able to predict the lift off as a nonreacting zone. 


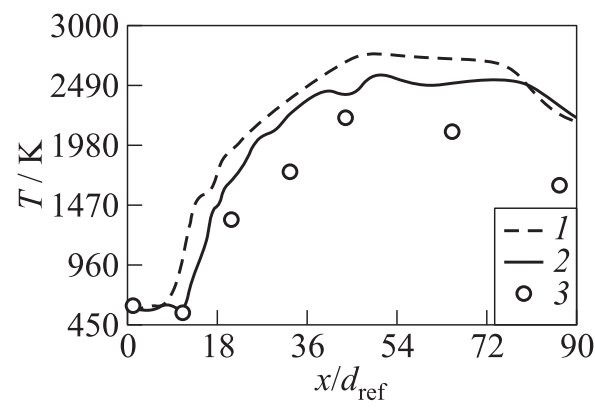

(a)

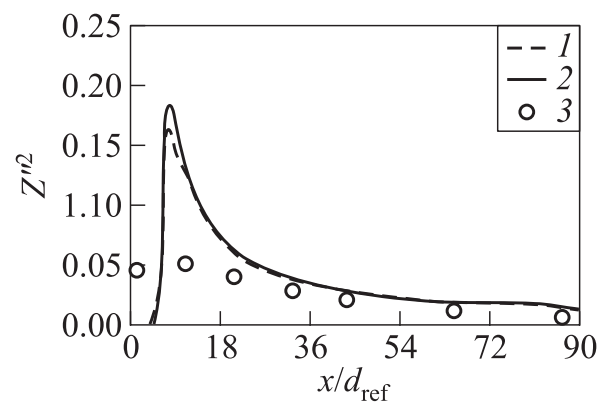

(c)

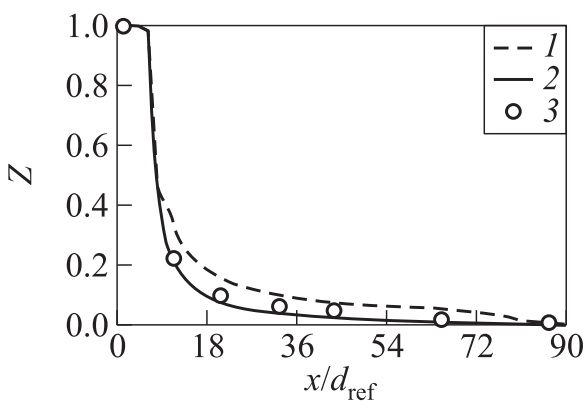

(b)

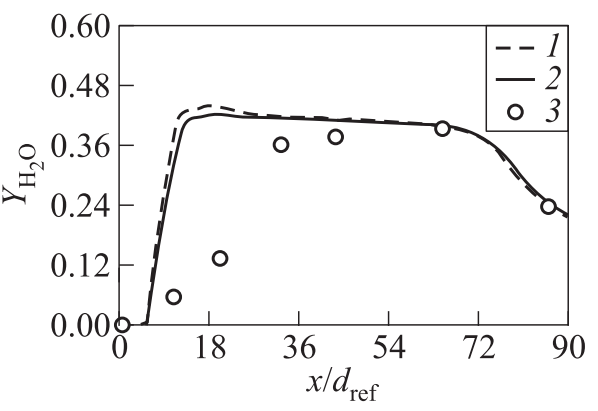

(d)

Figure 10 Cheng's flame thermochemical distribution taken along the axis $\left(y / d_{\text {ref }}\right.$ $=0): 1-$ model $\mathrm{A} ; 2$ - model $\mathrm{C}$; and 3 - experimental data [8]

Figure 10 reports the temperature, mixture fraction mean and variance, and water mass fraction distributions taken on the axis, namely, at $y / d_{\text {ref }}=0$. It appears that the both models are not able to properly reproduce the flame core; indeed, both provide a too high reaction rate so that the reactions may occur wherever the two flows $\left(\mathrm{H}_{2}-\right.$ wet air $)$ are mixing. As a consequence, models $\mathrm{A}$ and $\mathrm{C}$ predict the $Y_{\mathrm{H}_{2} \mathrm{O}}$ maximum value at about $10 x / d_{\text {ref }}$ as shown in Fig. $10 d$; the temperature distribution obtained with model $\mathrm{C}$ presents a slightly better agreement with experimental data [8] (see Fig. 10a).

Figure 11 shows distributions of such quantities taken at three radial sections, namely, $x / d_{\text {ref }}=0.85,10.8$, and 43.1 . In spite of the barely acceptable prediction of the flame core that is reflected in the temperature and water mass fraction spike in the first section of the near-axis region, here, one finds a good agreement with experimental data [8] with both models. Both the flame length and the mixture fraction consumption are well predicted, with the model $\mathrm{C}$ slightly outperforming model A (see Figs. 10 and 11). 

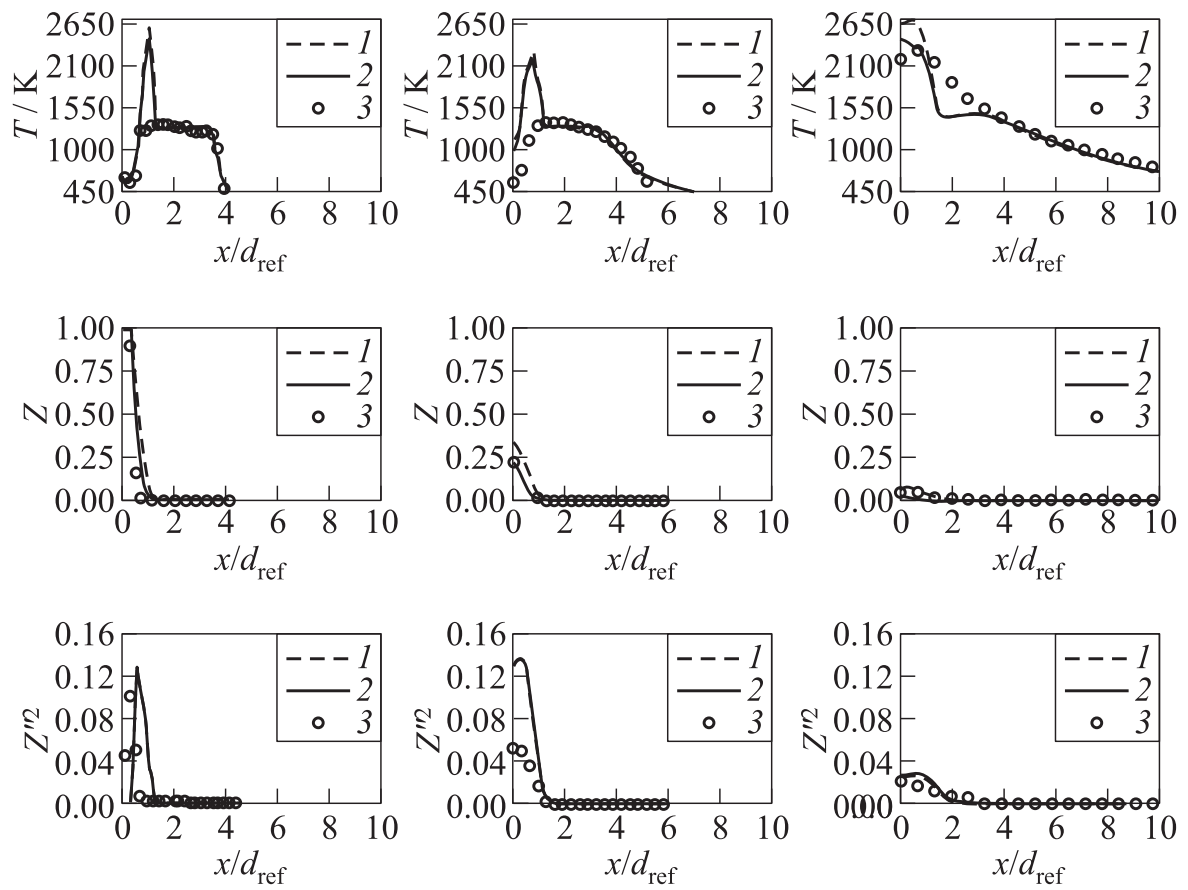

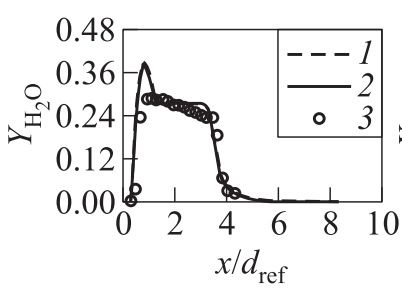

(a)

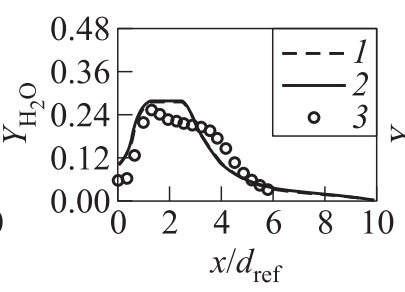

(b)

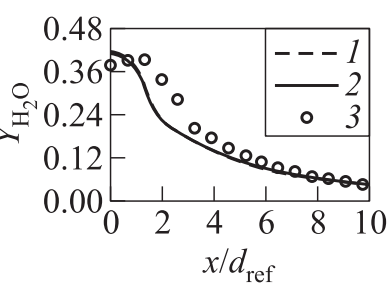

(c)

Figure 11 Cheng's flame thermochemical distribution taken at $x / d_{\text {ref }}=0.85(a)$, $10.8(b)$, and $33.1(c)$, respectively: 1 - model A; 2 - model C; and 3- experimental data $[8]$

\section{CONCLUDING REMARKS}

This paper provides an extension of standard FPV model combined with a RANS solver introducing the SMLD approach to describe the progress variable distribution. The work is composed of four sections. The first one is an introduction to the problem of the presumed PDFs in nonpremixed combustion. The 
second section describes the combustion model developed with a new closure method for the SMLD technique. The third section provides the flow governing equations, the additional transport equations of the combustion models, and the main features of the numerical method. It is noteworthy to observe that the analytical form proposed for the $P_{\mathrm{SML}, 2}$, and the particular form of the Lagrangian multipliers, does not introduce any additional computational effort with respect to that required to model the progress variable distribution as a $\beta$ function. In fact, one has to solve the same number of equations for the combustion model. These two approaches are both slightly more expensive than the standard one, since an additional transport equation has to be considered and a four-dimensional flamelet library has to be built and stored instead of a three-dimensional one. Moreover, it is interesting to observe that the proposed model represents a more general framework built relaxing the hypothesis about the shape of $P(C)$.

The results are discussed in the last section for the case of a subsonic $\mathrm{CH}_{4}-$ air flame and an $\mathrm{H}_{2}$-air Mach 2 flame. The analysis is performed in order to validate the applicability of the developed models. In the first case, the flow is simple and the steady flamelet equation reproduces the phenomenon adequately, providing a good agreement with the experimental data. In the second case, although some of the assumptions of the FPV model are not fulfilled, the numerical results can be considered satisfactory; the models predict a flame completely attached to the burner probably due to the missing correlation between the mixture fraction and the progress variable that are considered statistically independent.

\section{REFERENCES}

1. Maas, U., and S. Pope. 1992. Simplifying chemical kinetics-intrinsic lowdimensional manifolds in composition space. Combust. Flame 88:239-264.

2. Pierce, C. 2001. Progress-variable approach for large-eddy simulation of turbulent combustion. Stanford University. PhD Thesis.

3. Gicquel, O., N. Darabiha, and D. Thevenin. 2000. Laminar premixed hydrogen/air counterflow flame simulations using flame prolongation of ILDM with differential diffusion. Proc. Combust. Inst. 28:1901-1908.

4. Oijen, J. V., and L.D. Goey. 2000. Modelling of premixed laminar flames using flamelet-generated manifolds. Combust. Sci. Technol. 161:113-137.

5. Peters, N. 2000. Turbulent combustion. Cambridge University Press. 170-193.

6. Pope, S. 1985. PDF methods for turbulent reactive flows. Prog. Energy Combust. Sci. 11:119-192.

7. Sandia National Laboratories, TNF Workshop. Available at: http://www.ca. sandia.gov/TNF (accessed June 22, 2015). 
8. Cheng, T.S., J. A. Wehrmeyer, and R.W. Pitz. 1992. Simultaneous temperature and multispecies measurement in a lifted hydrogen diffusion flame. Combust. Flame 91(3):323-345.

9. Cutrone, L., P. De Palma, G. Pascazio, and M. Napolitano. 2010. A RANS flameletprogress-variable method for computing reacting flows of real-gas mixtures. Comput. Fluids 39:485-498.

10. Cook, A. W., and J. J. Riley. 1994. A subgrid model for equilibrium chemistry in turbulent flows. Phys. Fluids 6:2868-2870.

11. Jimenez, J., A. Linan, M. M. Rogers, and F. J. Higuera. 1997. A priori testing of subgrid models for chemically reacting non-premixed turbulent shear flows. J. Fluid Mech. 349:149-171.

12. Wall, C., B. J. Boersma, and P. Moin. 2000. An evaluation of the assumed beta probability density function subgridscale model for large eddy simulation of nonpremixed, turbulent combustion with heat release. Phys. Fluids 12:2522-2529.

13. Ihme, M., and H. Pitsch. 2008. Prediction of extinction and re-ignition in nonpremixed turbulent flames using a flamelet progress variable model. 1. A priori study and presumed PDF. Combust. Flame 155:70-89.

14. Heinz, S. 2003. Statistical mechanics of turbulent flows. Springer-Verlag. 19-23.

15. Shannon, C. H. 1948. A mathematical theory of communication. Bell Syst. Techn. J. $27: 379,423$.

16. Ihme, M., and H. Pitsch. 2008. Prediction of extinction and re-ignition in nonpremixed turbulent flames using a flamelet progress variable model. 2 Application in LES of Sandia Flames D and E. Combust. Flame 155:90-107.

17. Schwer, D. A. 1999. Numerical study of unsteadiness in non-reacting and reacting mixing layers. The Pennsylvania State University. PhD Thesis.

18. Steger, J. L., and R.F. Warming. 1981. Flux vector splitting of the inviscid gasdynamic equations with applications to finite difference methods. J. Comput. Phys. 40:263-293.

19. Pulliam, T. H., and D.S. Chaussee. 1981. A diagonal form of an implicit factorization algorithm. J. Comput. Phys. 39:347-363.

20. Buelow, P. E. O., D. A. Schwer, J.-Z. Feng, C. L. Merkle, and D. Choi. 1997. A preconditioned dual time diagonalized ADI scheme for unsteady computations. AIAA Proceedings. 120-130.

21. Menter, F., and C. Rumsey. 1994. Assessment of two-equation turbulence models for transonic flows. 25th AIAA Fluid Dynamics Conference. Colorado Springs, CO: AIAA.

22. Pitsch, H. Flamemaster v3.3. A $\mathrm{C}++$ computer program for 0d combustion and 1D laminar ame calculations.

23. Smith, G. P., D. M. Golden, M. Frenklach, N. W. Moriarty, B. Eiteneer, M. Goldenberg, C. T. Bowman, R. K. Hanson, S. Song, W. C. Gardiner, V. V. Lissianski, and Z. Qin. 2000. GRI-Mech 3.0. Available at: http://www.me.berkeley.edu/gri_mech/ (accessed June 22, 2015).

24. Gerlinger, P. 2007. Study of a multi-variate-beta-PDF for species distributions and model improvement. Technical Report LAPCAT Del. No. 6.6.4. 
25. Jarret, O. J., A.D. Cutler, R.R. Antcliff, T. Chitsomboon, C. L. Dancey, and J. A. Wang. 1988. Measurements of temperature, density, and velocity in supersonic reacting flow for CFD code validation. 25th JANAF Combustion Meeting. CPIA publications 498. 1:357-364.

26. Vajda, S., H. Rabitz, and A. Yetter. 1990. Effects of thermal coupling and diffusion on the mechanism of $\mathrm{H}_{2}$ oxidation in steady premixed laminar flames. Combust. Flame 82:270-297.

27. Pecnik, R., V.E. Terrapon, F. Ham, G. Iaccarino, and H. Pitsch. 2012. Reynoldsaveraged Navier-Stokes simulations of the HyShot II scramjet. AIAA J. 50(8):17171732 . 